\title{
The high cost of swing leg circumduction during human walking
}

\section{Authors:}

K. Alex Shorter ${ }^{\mathrm{a}}$ (Corresponding Author)

Email: kshorter@umich.edu

Amy $\mathrm{Wu}^{\mathrm{a}}$

Email: amyrwu@umich.edu

Arthur D. Kuo a,b

Email: artkuo@umich.edu

\author{
Affiliations: \\ ${ }^{a}$ Department of Mechanical Engineering \\ University of Michigan \\ G.G. Brown Laboratory \\ 2350 Hayward \\ Ann Arbor, MI 48109 \\ USA \\ ${ }^{\mathrm{b}}$ Faculty of Kinesiology \\ University of Calgary \\ Calgary, AB T2N 1N4 CANADA
}

\section{Acknowledgments:}

The authors would like to thank Genevieve Flaspohler, Meghna Menon and Sara Rusignuolo for their help with the work. This work was supported in part by the Office of Naval Research (ETOWL program), National Institutes of Health (AG030815), and Department of Defense (W81XWH-09-2-0142, National Defense Science \& Engineering Graduate Fellowship Program). 


\section{ABSTRACT}

2 Humans tend to walk economically, with preferred step width and length corresponding to an 3 energetic optimum. In the case of step width, it is costlier to walk with either wider or narrower

4 steps than normally preferred. Wider steps require more mechanical work to redirect the body's 5 motion laterally with each step, but the cost for narrower steps remains unexplained. Here we 6 show that narrow steps are costly because they require the swing leg to be circumducted around 7 the stance leg. Healthy adults $(\mathrm{N}=8)$ were tested walking with varying levels of circumduction, 8 induced through lightweight, physical obstructions ("Fins") attached medially to the lower legs, 9 during treadmill walking at fixed speed $\left(1.25 \mathrm{~m} \cdot \mathrm{s}^{-1}\right)$ and step width. The net rate of metabolic 10 energy expenditure increased approximately with the square of circumduction amplitude, by 11 about 50\% for an amplitude (measured at mid-swing) of about $18 \mathrm{~cm}$. Subjects also generated 12 greater stance leg torque and more arm motion to counter the circumduction, among other 13 compensatory motions that may contribute to energy expenditure. The costs of producing and 14 countering lateral leg motion partially explains the poorer economy of some gait pathologies 15 where circumduction may occur, for example stiff-knee gait. And for healthy individuals, it 16 explains how the energetically optimal average step width, along with the additional variability 17 inherent with multiple steps, should be narrow enough to avoid excessive redirection of the body, 18 yet wide enough to avoid costly circumduction. Humans appear to prefer a step width that 19 compromises between the competing energetic costs for either wider or narrower steps.

21 Key Words: Biomechanics, Gait, Circumduction, Energetics, Locomotion 


\section{INTRODUCTION}

2 The swing foot's motion during walking follows a fairly straight path when viewed from above.

3 Mirroring paths of the opposing feet seem to be separated by just enough distance to allow

4 movement past each other without colliding. A narrower step width would require lateral

5 circumduction of the swing leg to avoid the stance foot. However, such circumduction might

6 require additional effort, as any healthy person can experience with a few minutes' practice. Gait

7 pathologies such as foot drop or stiff-knee gait often lead to circumduction and other

8 compensations that gain better swing leg ground clearance, but at considerably greater energy

9 expenditure (Waters and Mulroy, 1999). Part of the added cost is likely attributable to

10 circumduction itself, in amount both unknown and difficult to isolate from other contributions.

11 Even for the healthy, it remains unproven whether the normal swing foot path is chosen to avoid

12 circumduction. Quantification of the energetic cost of circumduction might lend insight

13 regarding the swing leg motion in both healthy and pathological gait.

14 Normal step width may be determined by two opposing trade-offs: a cost for wider steps and a

15 cost for circumduction (Donelan et al., 2001). Wider steps entail more side-to-side motion of the

16 body center of mass (COM). Simple models show that redirecting motion requires mechanical

17 work - termed step-to-step transition work-increasing with the square of step width (Kuo,

18 1999), as supported by experimental data for both work and a proportional metabolic cost

19 (Donelan et al., 2001). But step-to-step transition costs are minimized at zero step width, whereas

20 the observed minimum for humans is at the preferred width of about $14 \mathrm{~cm}$ (midline-to-midline

21 of the feet), just wide enough for the feet to pass each other without circumduction. This suggests

22 an additional cost for narrower steps, explained not by step-to-step transitions, but perhaps by the

23 need to circumduct the swing leg around the stance leg.

24 There are several reasons why swing leg circumduction might be costly. There may be a cost

25 directly attributable to muscular effort needed to deviate the leg laterally during swing. The

26 passive dynamics of the thigh and shank act like coupled pendulums (Dean and Kuo, 2009;

27 McGeer, 1990; Mochon and McMahon, 1980), with natural motion that is largely planar.

28 Muscular force and work would presumably be needed to deviate from the sagittal plane. But

29 there may also be other, more indirect costs. To produce circumduction, the body must also

30 create opposing reaction forces, which must be transmitted either to the ground or to opposing 
1 motions of other limbs. This is similar to how the arm swing of normal walking appears to react

2 against the vertical twisting induced by the legs (Elftman, 1939), resulting in higher energy cost

3 when walking without arm swing (Collins et al., 2009).

4 The purpose of this study is to quantify the mechanical and metabolic costs of swing leg

5 circumduction. We hypothesize that increased circumduction will result in greater metabolic 6 energy expenditure. That cost should be associated with the direct actions producing 7 circumduction and indirect reactions in the stance leg and arms. We performed an experiment 8 using physical constraints to induce varying amounts of circumduction in healthy adults, similar 9 to a preliminary experiment reported previously (Shipman et al., 2002). We expected energy cost

10 to increase with greater swing leg circumduction and lead to compensatory actions throughout 11 the arms, and stance leg, with these added indirect costs contributing to greater metabolic cost 12 during walking.

\section{EXPERIMENTAL METHODS}

14 We measured energetic and mechanical effects of walking with artificially induced swing leg 15 circumduction in healthy adults. The amount of circumduction was modulated using lightweight 16 foam obstacles - "Fins" - on the medial aspect of each leg to interfere with normal swing

17 (Figure 1). Subjects were asked to walk with circumduction to avoid collisions between the Fins, 18 while being constrained to their preferred step width. For comparison, subjects were also asked 19 to walk with zero step width. Measurements included swing foot trajectories as an indicator of 20 circumduction, the associated energy expenditure, and the amount of arm swing and ground 21 reaction moments as indicators of indirect reactions to circumduction.

22 Eight healthy young adults (5 males and 3 females; body mass $M$ of $73 \pm 13 \mathrm{~kg}$, mean \pm s.d; leg 23 length $L$ of $0.89 \pm 0.08 \mathrm{~m}$ ) participated in the study, walking on an instrumented treadmill at 1.25 $24 \mathrm{~m} \cdot \mathrm{s}^{-1}$ in six experimental conditions. Fins were strapped medially to the shank of each leg, 25 with different horizontal widths requiring greater circumduction (Figure 2). The Fins were in five 26 widths ranging 0-127 mm (labeled Fin0, Fin2, Fin3, Fin4, and Fin5, numbered according to the 27 width in inches) and approximately weight-matched (about $15 \mathrm{~g}$, closed-cell foam $13 \mathrm{~mm}$ thick). 28 The sixth experimental condition required the subject to walk with zero step width (with Fin0 for 
1 weight matching). All subjects provided written informed consent prior to the study in 2 accordance with Institutional Review Board procedures.

3 Step width was controlled in each condition. The subject's preferred step width was determined 4 during 6 minutes of normal, unconstrained treadmill walking at $1.25 \mathrm{~m} / \mathrm{s}$. That same width 5 (about $0.18 \pm 0.02 \mathrm{~m}$, mean \pm s.d.) was used for the subject's five Fin trials, displayed as a pair 6 of laser lines projected longitudinally onto the treadmill. Subjects were instructed to step on the 7 laser lines, aided by a real-time video projection of their feet and the lines, displayed in front of 8 them. For the zero step width condition, subjects were asked to step on a single laser line.

9 Data collection was performed as follows. Oxygen consumption and carbon dioxide production 10 were measured using a respirometry system (CareFusion, Vernon Hills, IL, USA). Steady state 11 energy expenditure was calculated from the final 3 minutes of each 6 minute trial. Gross 12 metabolic energy expenditure (in W) was calculated using standard conversion factors 13 (Brockway, 1987), and the rate for quiet standing (112 $\pm 19 \mathrm{~W})$ subtracted to yield net metabolic 14 rate. Also measured were ground reaction forces (instrumented treadmill, Bertec, Columbus, OH, 15 USA) and lower extremity kinematics (motion capture, Phasespace, San Leandro, CA, USA), for 16 one minute of each trial, and used for standard inverse dynamics analysis (Visual3D, C-Motion, 17 Germantown, MD, USA). Additional kinematics were recorded throughout the entire trial from 18 inertial measurement units (APDM Inc, OR, USA), secured to the insteps of both shoes, and to 19 both wrists. The IMUs recorded acceleration and angular velocity of each foot, which were then 20 used to compute estimate of foot trajectories, using previously validated integration methods 21 (Ojeda and Borenstein, 2007; Rebula et al., 2013).

22 These data were processed to yield measures of circumduction and associated gait 23 compensations. Heel strike events were identified from ground reaction forces (Fin trials) and 24 kinematic data (zero step width trial). Circumduction was measured laterally from the straight 25 line connecting consecutive heel strikes, sampled at mid-swing (a consistent gait event defined at $2680 \%$ of a stride cycle), near the time of maximum lateral excursion. Energetic cost was 27 quantified with net metabolic rate, and indirect compensations with the peak stance leg moment 28 (free vertical ground reaction moment) and the peak arm acceleration (from translational 29 accelerations of wrist IMUs). Effects on gait parameters were quantified with step length and 
1 width from heel marker positions sampled at mid-stance, and step period and double support

2 duration (from ground reaction forces). To account for differences in body size, measurements

3 were non-dimensionalized using base units of body mass $M$, standing leg length $L$ (ground to

4 greater trochanter), and gravitational acceleration $g$. The mean normalization constant for step

5 length and step width was $L$ (average $0.89 \mathrm{~m}$ ), step time $\sqrt{L / g}(0.30 \mathrm{~s})$, force $M g(741 \mathrm{~N})$

6 moment $M g L(642 \mathrm{~N}-\mathrm{m})$, and power $M g^{1.5} L^{0.5}(2121 \mathrm{~W})$.

7 We performed statistical tests or differences in circumduction, energy cost, and other variables.

8 Paired t-tests were used to test discrete fin conditions against the Fin0 condition, and linear

9 regression for dependence on mid-swing circumduction (significance level $\alpha=0.05$ ). For

10 metabolic rate, we tested for a dependence on the square of the circumduction amplitude,

11 expected because the mechanical work required to move the leg from side to side should increase

12 thus (similar to back-and-forth; Doke et al., 2005).

\section{RESULTS}

14 The fin obstacles induced significant changes in circumduction (see Figure 2, Table 1), resulting

15 in a controlled, graded effect on limb circumduction $\left(P<10^{-4}\right.$ paired post-hoc t-test with Holm-

16 Sidak correction), with an amplitude of about $16 \mathrm{~cm}$ with the largest fins. Metabolic cost

17 increased with greater circumduction (Figure 3a), about $60 \%$ greater for Fin5 than Fin0, and

18 changing approximately with the square of mid-swing circumduction. There were also indirect

19 biomechanical changes, such as greater torque applied by the stance leg to the ground (i.e., free

20 vertical moment, Figure 3b). Arm swing also increased, indicated by increasing peak

21 translational acceleration of the swinging wrist (Figure 3c).

22 There were also small but significant effects on average step period, step length, and step width 23 both in terms of discrete conditions $\left(P<10^{-4}, P=1.1 \mathrm{e}-3, \mathrm{P}=4.2 \mathrm{e}-3\right.$, respectively; paired t-test,

24 Table 1) and with circumduction as a continuous regression variable. Contrary to both instruction 25 and the step width constraint, the subjects slightly increased their step width as the fins grew 26 larger (Figure 4A). Step length and step period also increased while double support duration 27 decreased, all linearly with circumduction (Figure 4).

28 The Zero step width condition also had effects on gait (Table 1). Most notably, this condition 29 resulted in significantly more circumduction than the Fin0 condition $\left(P<10^{-4}\right.$, paired t-test), and 
1 roughly comparable to the Fin4 and Fin5 conditions (Figures 5a and b). Metabolic rate also

2 increased by about $28 \%$ compared to Fin0, similar to the Fin 4 condition.

\section{DISCUSSION}

4 We had sought to systematically investigate the energetic cost of swing leg circumduction. We

5 found increasing cost with greater circumduction, attributable in part to direct costs for moving

6 the swing leg. However, we also observed increasing indirect effects associated with

7 compensatory strategies such as stance leg ground reaction moment and arm acceleration, which

8 may also contribute to the cost of walking with narrow steps. We propose that swing leg

9 circumduction may play a role in the selection of preferred step width, particularly in relation to

10 lateral balance control.

11 Energy cost and biomechanical variables were minimized for unobstructed walking (Figure 3).

12 The greater metabolic rate could potentially be explained by the work needed to circumduct the

13 leg. Mechanical work increases with the square of amplitude for periodic leg swinging (Doke et

14 al., 2005). Applying similar pendulum-like mechanics to lateral motion, work should increase

15 with the square of circumduction amplitude (with step length and period remaining relatively

16 unchanged, Table 1). However, the present study cannot exclude alternative explanations for the

17 cost of greater circumduction.

18 We also observed indirect effects from circumduction. Circumduction of the swing leg requires

19 an equal and opposite reaction forces and moments, which were transmitted to the ground (e.g.,

20 free vertical moment, Figure $3 b$ ) or into accelerations of other body segments such as the arms

21 (e.g., Figure 3c), consistent with previous observations that walking while preventing arm

22 swinging results greater free vertical moment and about 7\% greater energetic cost (Collins et al.,

23 2009). Here, subjects tended to swing their arms more, and these and other compensations

24 throughout the body may have required greater muscle effort and therefore contributed to

25 energetic cost.

26 Walking with narrow steps requires circumduction of the swing leg to avoid collision with the

27 stance leg. This is illustrated by the zero step width condition, where circumduction $(0.12 \pm 0.02$

$28 \mathrm{~m}$ ) exceeded that for Fin0, and was comparable to the Fin4 and Fin5 conditions (Table 1 and 
1 Figures 5 A \& B). Thus, costs observed from experimentally-induced circumduction suggest a 2 substantial contribution to the cost of walking with narrow steps.

3 We next return to the original hypothesis that the preferred step width may result from the trade4 off between the costs for circumduction and for redirecting the COM during step-to-step 5 transitions (Donelan et al., 2001; see Fig. 5C). The step-to-step transition cost $P_{\mathrm{s}-\mathrm{s}}$ increases 6 with the square of step width (Kuo, 1999), thus favoring zero width if not for $P_{\text {circ }}$, the 7 hypothesized cost for circumduction. Indeed, the preferred step width observed here appears to 8 be just wide enough to afford nearly zero circumduction $(0.01 \pm 0.02 \mathrm{~m}$ for Fin0 condition, 9 Figure 5a). Here we also refine the circumduction hypothesis to factor in the role of foot 10 placement variability (Fig. 5C inset), which should also affect the average cost of a given step 11 width. This is because some steps will vary medially and require more circumduction, while 12 others will vary laterally and exact a higher step-to-step transition cost. The overall effect is 13 summarized by the expected value function $E[\cdot]$,

$$
E\left[P_{n e t}(w)\right]=\int_{-\infty}^{\infty}\left(P_{\text {circ }}(w+\tau)+P_{\text {s-s }}(w+\tau)\right) p(\tau) d \tau
$$

14 where $w$ denotes mean step width, $p$ the associated variability about the mean (as a probability 15 distribution), and $P_{\text {circ }}, P_{\mathrm{s}-\mathrm{s}}$, and $P_{\text {net }}$ respectively denote circumduction, step-to-step transition, 16 and net contributions to the metabolic power associated with step width. If circumduction is 17 relatively costly compared to the cost of taking a wider and variable steps, it may be 18 economically favorable overall to take slightly wider steps on average, despite their greater step19 to-step transition cost.

20 This statistical approach can help explain some observed effects of external lateral stabilization. 21 Humans walking (on a treadmill) with their bodies laterally stabilized by elastic cords, decrease 22 their lateral foot placement variability as well their preferred step width (Donelan et al., 2004). 23 External stabilization decreases the need for active foot placement for balance, and therefore 24 explains the decreased variability, but not (directly) the preferred step width. The expected cost 25 above explains how decreased variability should favor a narrower mean step width due to less 26 likelihood for the swing leg to collide with the stance leg. An additional observation is that older 27 adults have greater step variability, prefer wider steps than young, and have less of a difference 
1 with external stabilization (Dean et al., 2007). If circumduction is relatively costly compared to 2 taking a wider step, older adults might again be expected to compensate for the greater 3 probability of experiencing a costly narrow step (higher step variability) by increasing their step 4 width accordingly.

5 Our findings may have implications for impaired gait. Patients with stiff-kneed gait (e.g., from 6 stroke or cerebral palsy, Sutherland et al., 1990) or other pathologies restricting knee flexion may 7 employ circumduction, among other compensations, to improve ground clearance (Kerrigan et 8 al., 1991; Wren et al., 2005). Our results show that such compensations are energetically costly 9 even for healthy individuals, as also observed with constrained knee flexion (Lewek et al., 2012).

10 Of course, impaired gait occurs at slower speeds and poses many other limitations that may 11 outweigh the effects seen here. There should nevertheless be mechanical consequences to 12 circumduction, whatever the origin.

13 The findings here are subject to a number of limitations. We imposed circumduction as part of a 14 controlled experiment, but this may not replicate other causes for circumduction, such as walking 15 with narrow steps. It was also possible for subjects to elect other compensation strategies, not 16 measured here. Additionally, other issues such as cognitive effort of visual feedback, or explicit 17 changes in step variability were not considered here. Although many complexities were not 18 considered, their effects do not appear large enough to negate the high cost of circumduction 19 observed in this simple experiment.

20 Walking with narrow steps typically entails circumduction of the swing leg. That circumduction

21 comes at an increased metabolic cost, not only to move the leg itself, but also for compensatory 22 motions elsewhere in the body. Circumduction appears to trade off against the separate cost for 23 taking wider steps. Together, the two contributions appear to determine the metabolically 24 optimal (and normally preferred) step width, and may explain why it is most economical to walk 25 with the feet just passing by each other, with little circumduction.

\section{Conflict of Interest Statement}

27 Conflicts of interest: none 


\section{References}

Brockway, J. M. (1987). Derivation of formulae used to calculate energy expenditure in man. Hum. Nutr. Clin. Nutr. 41, 463-471.

Collins, S. H., Adamczyk, P. G. and Kuo, A. D. (2009). Dynamic arm swinging in human walking. Proc. R. Soc. B Biol. Sci. 276, 3679-3688.

Dean, J. C. and Kuo, A. D. (2009). Elastic coupling of limb joints enables faster bipedal walking. J. R. Soc. Interface R. Soc. 6, 561-573.

Dean, J. C., Alexander, N. B. and Kuo, A. D. (2007). The effect of lateral stabilization on walking in young and old adults. IEEE Trans. Biomed. Eng. 54, 1919-1926.

Doke, J., Donelan, J. M. and Kuo, A. D. (2005). Mechanics and energetics of swinging the human leg. J. Exp. Biol. 208, 439-445.

Donelan, J. M., Kram, R. and Kuo, A. D. (2001). Mechanical and metabolic determinants of the preferred step width in human walking. Proc. R. Soc. Lond. B Biol. Sci. 268, 19851992.

Donelan, J. M. J. M., Shipman, D. W. D. W., Kram, R. and Kuo, A. D. A. D. (2004). Mechanical and metabolic requirements for active lateral stabilization in human walking. J. Biomech. 37, 827-835.

Elftman, H. O. (1939). The function of the arms in walking. Hum. Biol. 11, $529-535$.

Hof, A. L., Gazendam, M. G. J. and Sinke, W. E. (2005). The condition for dynamic stability. J. Biomech. 38, 1-8.

Kerrigan, D. C., Gronley, J. and Perry, J. (1991). Stiff-legged gait in spastic paresis. A study of quadriceps and hamstrings muscle activity. Am. J. Phys. Med. Rehabil. Assoc. Acad. Physiatr. 70, 294-300.

Kuo, A. D. (1999). Stabilization of lateral motion in passive dynamic walking. Int. J. Robot. Res. 18, 917-930.

Lewek, M. D., Osborn, A. J. and Wutzke, C. J. (2012). The influence of mechanically and physiologically imposed stiff-knee gait patterns on the energy cost of walking. Arch. Phys. Med. Rehabil. 93, 123-128.

McGeer, T. (1990). Passive dynamic walking. Int. J. Robot. Res. 9, 62-82.

Mochon, S. and McMahon, T. A. (1980). Ballistic walking. J. Biomech. 13, 49-57.

Ojeda, L. and Borenstein, J. (2007). Non-GPS navigation for security personnel and first responders. J. Navig. 60, 391. 
Rebula, J. R., Ojeda, L. V., Adamczyk, P. G. and Kuo, A. D. (2013). Measurement of foot placement and its variability with inertial sensors. Gait Posture 38, 974-980.

3 Shipman, D. W., Donelan, J. M., Kram, R. and Kuo, A. D. (2002). Metabolic cost of lateral leg swing in human walking. In Abstracts of the IV World Congress of Biomechanics, Calgary, Alberta, Canada.

Sulzer, J. S., Gordon, K. E., Dhaher, Y. Y., Peshkin, M. A. and Patton, J. L. (2010). Preswing Knee Flexion Assistance Is Coupled With Hip Abduction in People With StiffKnee Gait After Stroke. Stroke J. Cereb. Circ. 41, 1709-1714.

9 Sutherland, D. H., Santi, M. and Abel, M. F. (1990). Treatment of stiff-knee gait in cerebral palsy: a comparison by gait analysis of distal rectus femoris transfer versus proximal rectus release. J. Pediatr. Orthop. 10, 433-441.

Waters, R. L. and Mulroy, S. (1999). The energy expenditure of normal and pathologic gait. Gait Posture 9, 207-31.

Wren, T. A. L., Rethlefsen, S. and Kay, R. M. (2005). Prevalence of specific gait abnormalities in children with cerebral palsy: influence of cerebral palsy subtype, age, and previous surgery. J. Pediatr. Orthop. 25, 79-83. 


\section{TABLES}

Table 1 Experimental result by circumduction condition, Fin0 - Fin5, as well as Zero step width condition. Results as presented in SI units, as well as dimensionless (d'less) form.

\begin{tabular}{lcccccc}
\hline Measure & Fin0 & Fin2 & Fin3 & Fin4 & Fin5 & Zero \\
\hline $\begin{array}{l}\text { Mid-swing } \\
\text { Circumduction (m) }\end{array}$ & $0.01 \pm 0.02$ & $0.03 \pm 0.02$ & $0.08 \pm 0.02$ & $0.09 \pm 0.02$ & $0.16 \pm 0.04$ & $0.12 \pm 0.02$ \\
Mid-swing & & & & & & \\
Circumduction (d'less) & $0.01 \pm 0.02$ & $0.04 \pm 0.02$ & $0.09 \pm 0.03$ & $0.10 \pm 0.03$ & $0.18 \pm 0.05$ & $0.13 \pm 0.02$ \\
Step Length (m) & $0.69 \pm 0.04$ & $0.68 \pm 0.04$ & $0.69 \pm 0.04$ & $0.70 \pm 0.04$ & $0.74 \pm 0.05$ & $0.71 \pm 0.02$ \\
Step Length (d'less) & $0.78 \pm 0.04$ & $0.76 \pm 0.04$ & $0.77 \pm 0.04$ & $0.78 \pm 0.04$ & $0.83 \pm 0.06$ & $0.80 \pm 0.06$ \\
Step Width (m) & $0.21 \pm 0.02$ & $0.24 \pm 0.03$ & $0.24 \pm 0.04$ & $0.26 \pm 0.04$ & $0.28 \pm 0.05$ & $0.00 \pm 0.02$ \\
Step Width (d'less) & $0.24 \pm 0.03$ & $0.27 \pm 0.03$ & $0.28 \pm 0.04$ & $0.29 \pm 0.03$ & $0.32 \pm 0.05$ & $0.00 \pm 0.02$ \\
Step Period (s) & $0.55 \pm 0.03$ & $0.55 \pm 0.03$ & $0.55 \pm 0.04$ & $0.56 \pm 0.03$ & $0.60 \pm 0.05$ & $0.57 \pm 0.02$ \\
Step Period (d'less) & $1.84 \pm 0.06$ & $1.81 \pm 0.05$ & $1.83 \pm 0.07$ & $1.86 \pm 0.07$ & $1.98 \pm 0.12$ & $1.90 \pm 0.05$ \\
Net metabolic rate (W) & $203 \pm 44.6$ & $220 \pm 41.9$ & $240 \pm 55.7$ & $247 \pm 45.7$ & $324 \pm 77.0$ & $259 \pm 61.2$ \\
$\begin{array}{l}\text { Net metabolic rate } \\
\text { (d'less) }\end{array}$ & $0.10 \pm 0.02$ & $0.10 \pm 0.02$ & $0.11 \pm 0.02$ & $0.12 \pm 0.02$ & $0.15 \pm 0.03$ & $0.12 \pm 0.02$ \\
\hline
\end{tabular}




\section{FIGURES}

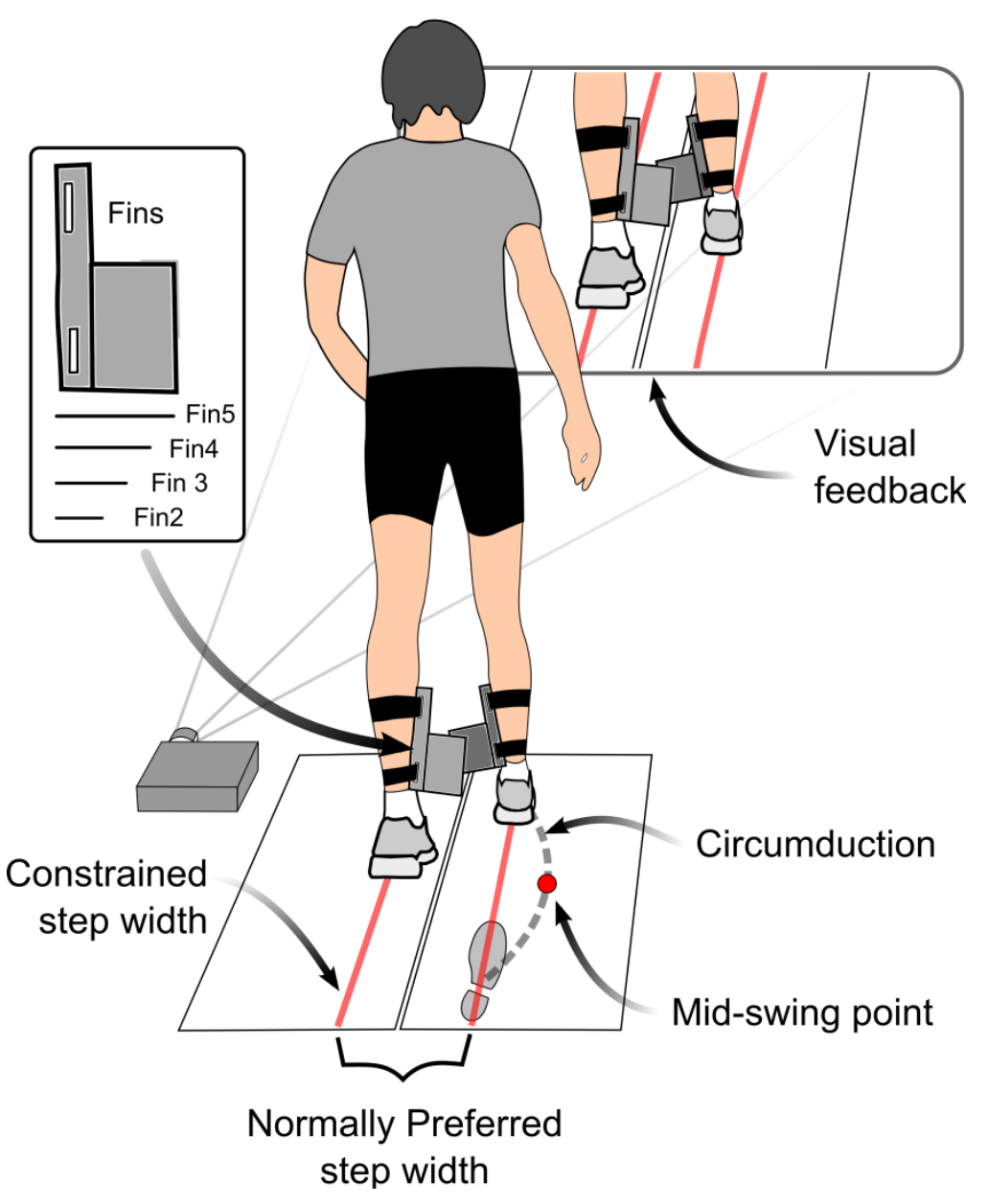

Figure 1 Setup for experimentally-induced swing leg circumduction. Circumduction was modulated using foam obstacles ("Fins") of various widths, secured medially to each shank. Step width was constrained using laser lines projected onto treadmill that subjects were asked to step on, with a projected video image as aid. 

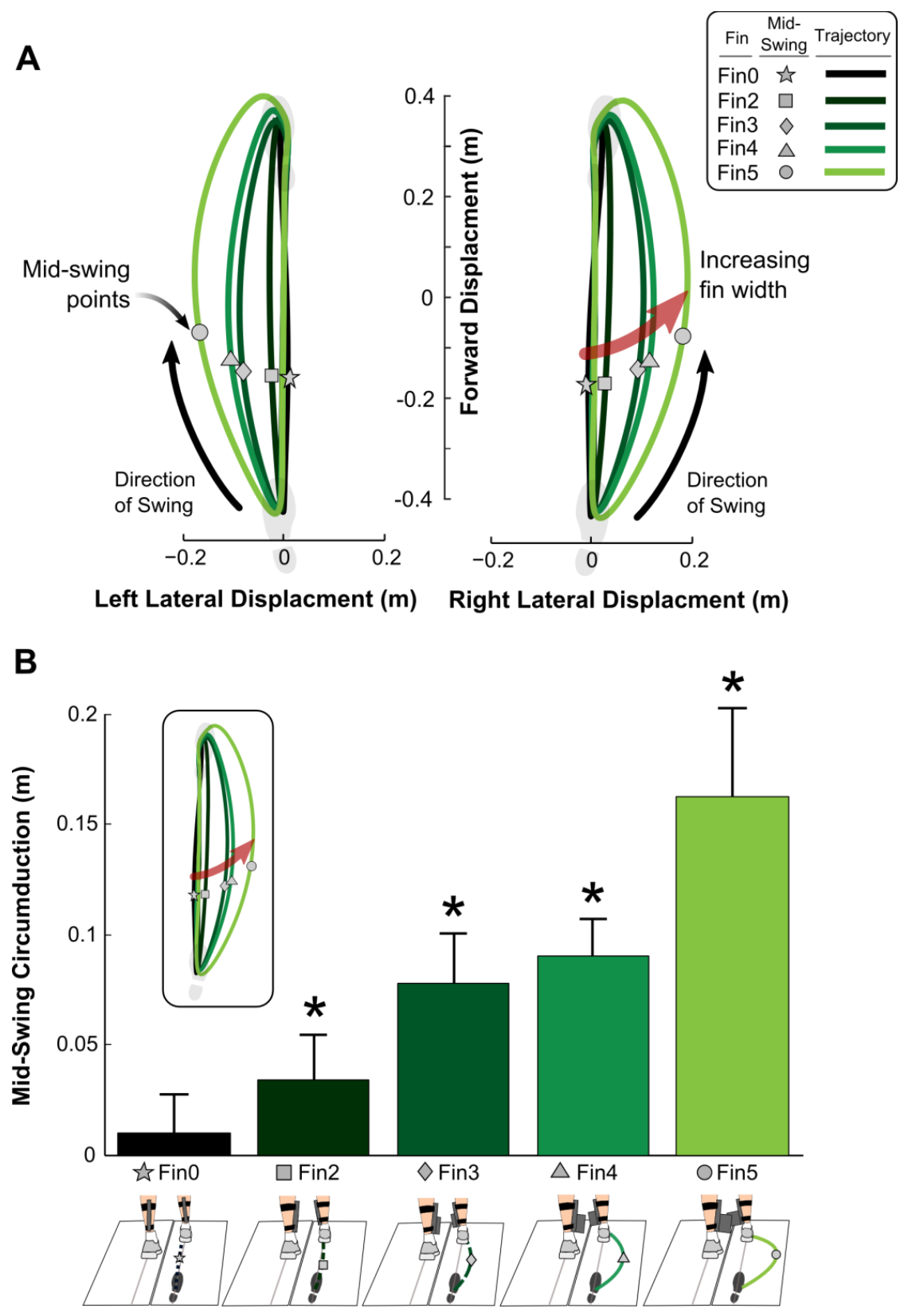

Fin Constraint

Figure 2 Effect of Fin constraints on swing foot trajectories. (A) Average foot trajectories (forward vs. lateral displacement of toe marker, in laboratory frame) for five Fin conditions. Mid-swing circumduction is measured as lateral deviation middle of swing (shaded symbols) for each trajectory at mid-swing. (B) Mid-Swing Circumduction (mean $\pm \mathrm{s}$. d. across subjects) vs. Fin condition. Asterisk * denotes significant difference from Fin0 condition. 

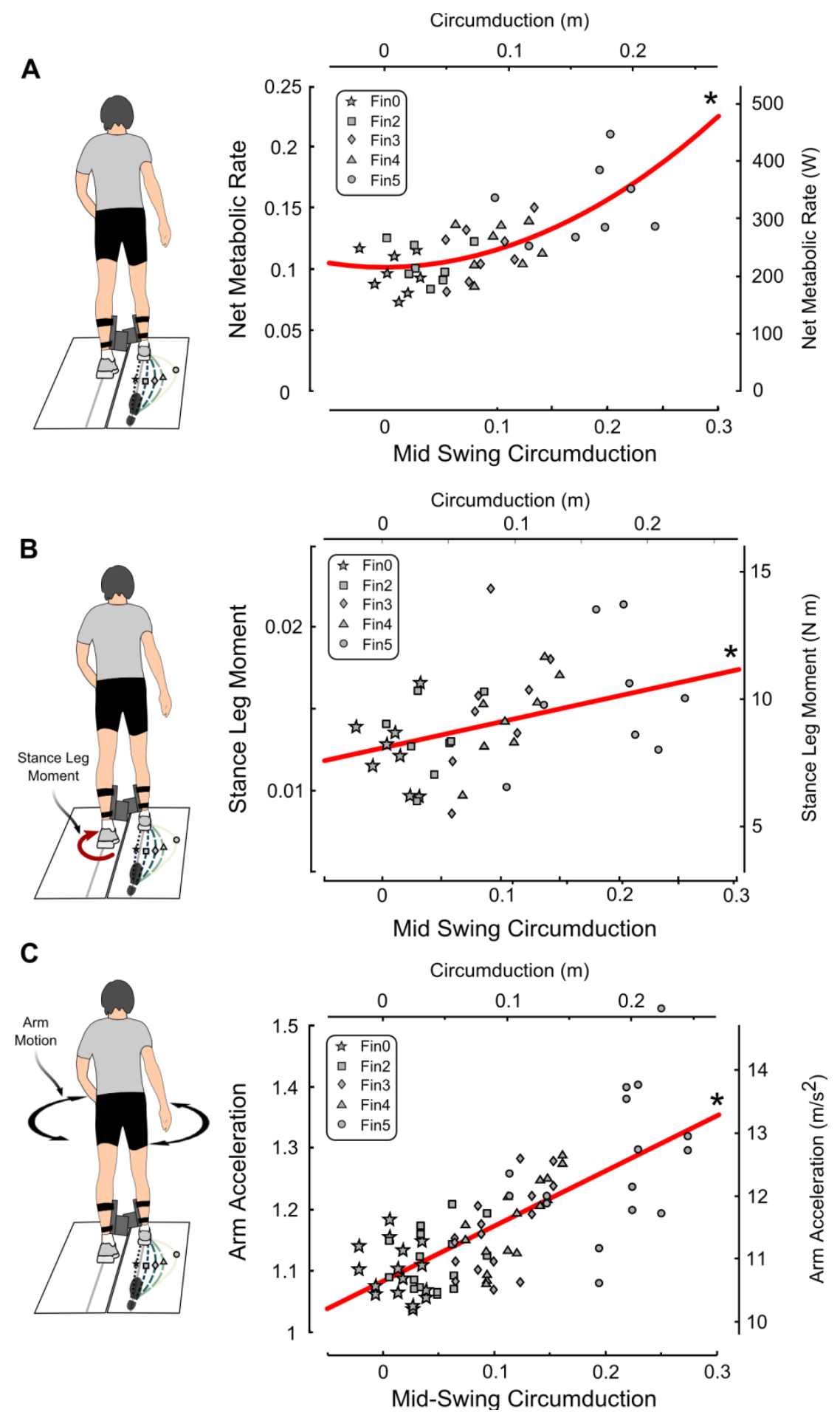

Figure 3 Energy expenditure and compensatory motions vs. mid-swing circumduction. (A) Net 4 metabolic rate data, with square fit $\left(1.36 x^{2}+0.1\right.$ where $x$ denotes circumduction; $\left.R^{2}=0.81\right)$. B) 5 Stance leg moment (peak of vertical free ground reaction moment) data, with linear fit $(0.2 x+$ $\left.60.01, R^{2}=0.32\right)$. C) Arm acceleration (peak) data, with linear fit $\left(0.89 x+1.09, R^{2}=0.66\right)$. Both 7 dimensionless (left and bottom axes) and SI units (right and top) are shown. Asterisk (*) 8 indicates significant regression. 

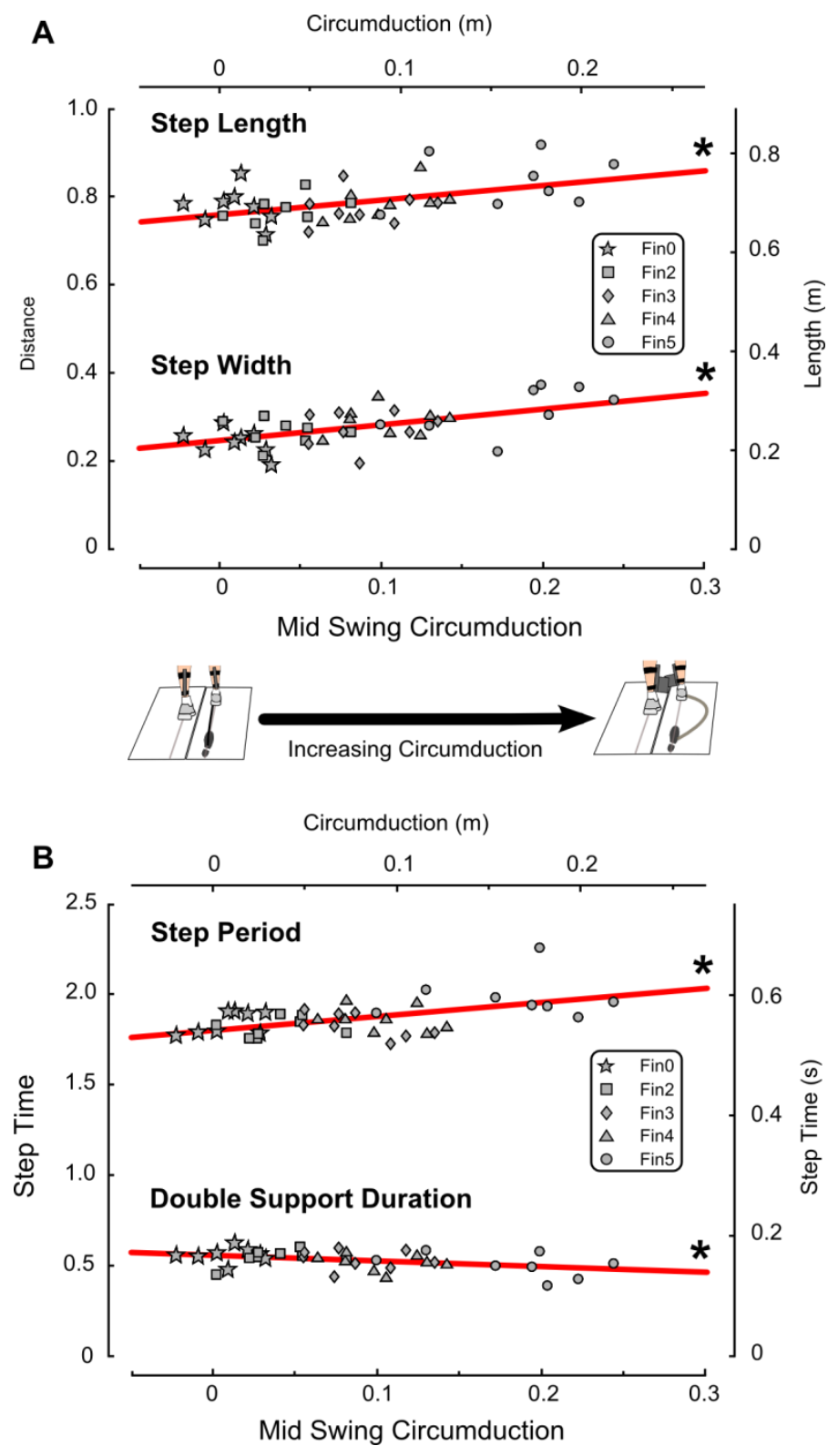

3 Figure 4 Step parameters as function of mid-swing circumduction, with linear fits. (A) Step 4 length $\left(0.33 x+0.76, R^{2}=0.55\right)$ and width data $\left(0.35 x+025 R^{2}=0.45\right)$ for all subjects. (B) 5 Step period $\left(0.76 x+1.80, R^{2}=0.51\right)$ and double support times $\left(-0.32 x+0.56, R^{2}=0.67\right)$ for 6 all subjects. Both dimensionless (left and bottom axes, using base units of body mass, leg length 7 and gravitational acceleration) and SI units (right and top) are shown. Asterisk (*) indicates 8 significant regression. 

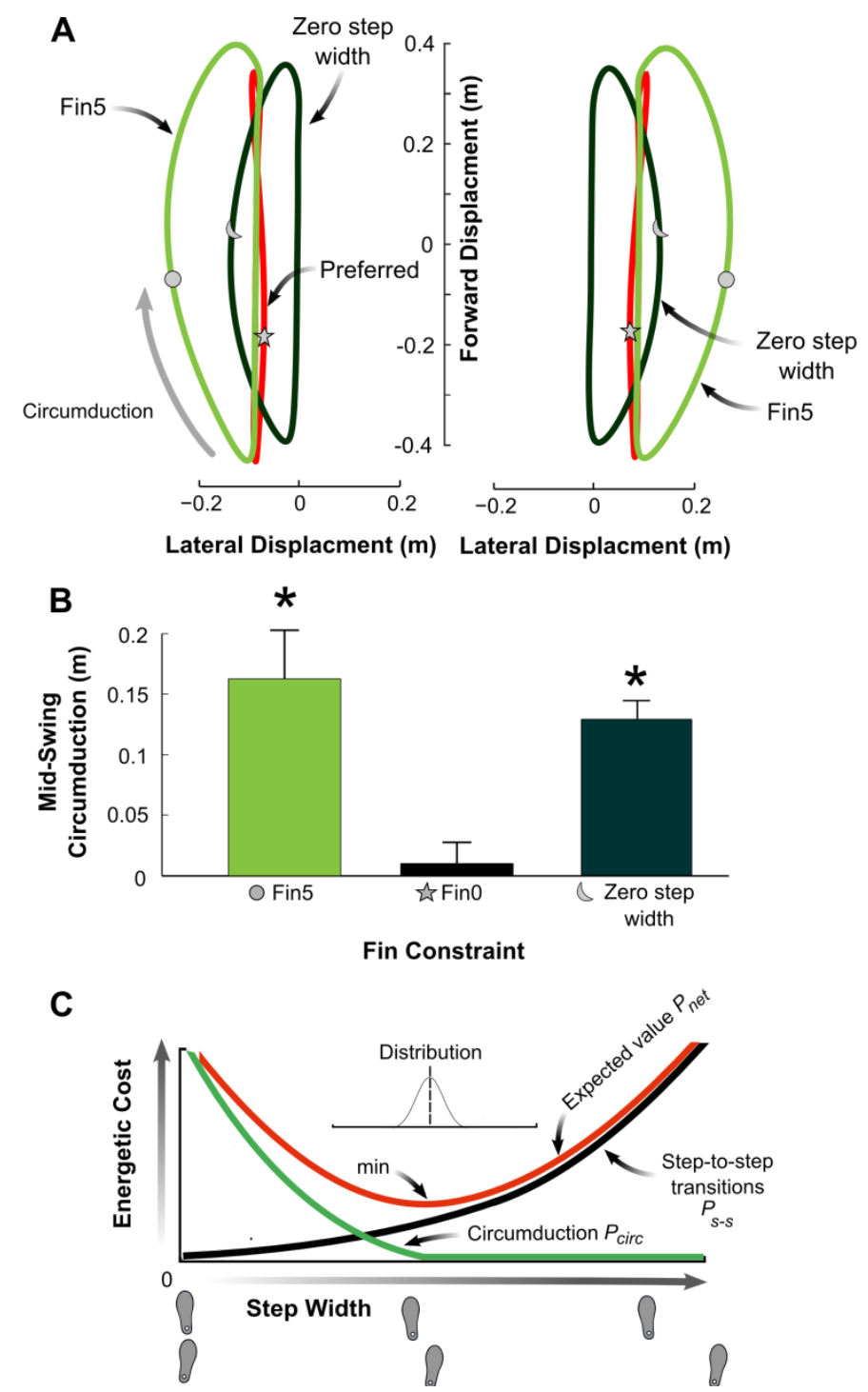

Figure 5 Interaction between swing leg circumduction and step width. (A) Foot trajectories for 4 the Fin0 and Fin5 conditions (at fixed step width) and the zero step width condition. (B) Mid5 Swing Circumduction (mean \pm s. d. across subjects) vs. condition. Asterisk (*) denotes 6 significant difference from Fin0 condition. (C) Conceptual illustration of how both wide and 7 narrow step widths contribute to metabolic cost. Wider steps entail step-to-step transition costs, $8 P_{\mathrm{s}-\mathrm{s}}$, increasing with step width squared. Narrower steps require increasing swing leg 9 circumduction costs, $P_{\text {circ }}$. The expected value of the sum of these two costs, $P_{\text {net }}$, incorporating step width variability (Inset), explains why an intermediate step width may be optimal. 